\section{SAÚDEE AMBIENTE}

V.7 $\cdot$ N.3 $\cdot$ Abril -2019

ISSN Digital: 2316-3798

ISSN Impresso: 2316-3313

DOI: $10.17564 / 2316-3798.2019 v 7 n 3 p 49-60$ ORCID: 0000-0002-7779-5126
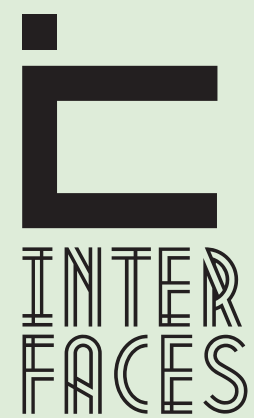

CIENTÍFICAS

\section{SUICÍDIO E POVOS INDÍGENAS NO BRASIL}

SUICIDE AND INDIGENOUS POPULATION IN BRAZIL

SUICIDIO Y POBLÁCION INDÍGENA EN BRASIL

Vilma Janaína Rios Cabral Victal ${ }^{1}$ Bianca Tenório de Aguiar ${ }^{2}$ Antônio Fernando Silva Xavier Júnior ${ }^{3}$ Cyro Rego Cabral Júnior ${ }^{4}$

\section{RESUMO}

Indígenas vivem menos, têm piores serviços de saúde e de educação e têm taxas de desemprego mais altas que a população em geral. As taxas de suicídio destas comunidades também superam muito as taxas da população em geral, mesmo em países desenvolvidos. 0 presente estudo buscou levantar os óbitos por suicídio no Brasil ocorridos de 2005 a 2014, a forma como se distribuem geograficamente, as taxas de mortalidade em cada etnia e as faixas etárias mais atingidas. Visou ainda determinar a associação entre etnia e mortalidade por suicídio. Estudo transversal das frequências absolutas de suicídio. Observou-se que o número de óbitos por suicídio entre indígenas foi inferior ao de quase todas as outras etnias; porém, a sua taxa de mortalidade por suicídio foi a mais alta. Houve associação significativa entre ser indígena e suicídio, tanto em $2005\left(X^{2}=174,9 ; p<0,001\right)$ quanto em $2014\left(X^{2}=304,6 ; p<0,001\right)$. No mesmo período, verificou-se que ser indígena consistiu-se em fator de risco para o suicídio (2005: OR $=4,04$; IC95\%=3,22-5,05 e 2014: OR =4,18; IC95\% = 3,51$4,98)$. A faixa etária mais atingida entre os indígenas brasileiros no período foi a de 15 a 19 anos, seguida pela faixa de 20 a 29 anos. 0 estado da federação que registrou o maior número de óbitos foi Mato Grosso do Sul, seguido pelo Amazonas. Os dados encontrados indicam que estas comunidades estão em situação perigosa, demandando, assim, a implementação de políticas de saúde mental que deverão priorizá-las, levar em conta suas peculiaridades e seu direito de autodeterminação.

\section{PALAVRAS-CHAVE}

Epidemiologia. Suicídio. População Indígena. Etnia. 


\section{ABSTRACT}

Indigenous people live less, have worse health and education services and have higher unemployment rates than the general population. The suicide rates of these communities also far exceed the rates of the general population, even in developed countries. The present study aimed to find out suicide deaths in Brazil from 2005 to 2014, it's geographical distribution, the mortality rates in each ethnic group and the most affected age groups. It also aimed to discover the association between ethnicity and suicide mortality. Cross-sectional study of absolute suicide rates. Data shows that the number of suicide deaths among natives was lower than that of almost all other ethnic groups; however, the suicide mortality rate was the highest. There was an association between being indigenous and suicide, both in $2005\left(X^{2}=174.9 ; p<0.001\right)$ and $2014\left(X^{2}=304.6 ; p<0.001\right)$. In the same period, being indigenous was a risk factor for suicide (2005: OR = 4.04; IC95\%=3.22-5.05 e 2014: OR =4.18; IC95\% $=3.51-4.98)$. The most affected age group among Brazilian natives in the period was 15 to 19 years, followed by the age range of 20 to 29 years. The state of the federation that recorded the highest number of deaths was Mato Grosso do Sul, followed by Amazonas. The data show that these communities are in a dangerous situation, thus demanding mental health policies that should prioritize them, take into account their peculiarities and their right to self-determination.

\section{KEYWORDS}

Epidemiology. Suicide. Indigenous Population. Ethnicity.

\section{RESUMEN}

Indígenas viven menos, tienen peores servicios de salud y de educación y tienen tasas de desempleo más altas que la población en general. Las tasas de suicidio de estas comunidades también superan mucho las tasas de la población en general, incluso en países desarrollados. El presente estudio busca levantar las muertes por suicidio en Brasil ocurridos de 2005 a 2014, la forma como se distribuyen geográficamente, las tasas de mortalidad en cada etnia y las franjas etarias más afectadas. En el presente estudio se evaluó la asociación entre etnia y mortalidad por suicidio. Estudio transversal de las frecuencias absolutas de suicidio. Se observó que el número de muertes por suicidio entre indígenas fue inferior al de casi todas las otras etnias: sin embargo, su tasa de mortalidad por suicidio fue la más alta. Hubo asociación entre ser indígena y suicidio, tanto en $2005\left(X^{2}=174,9 ; p<0,001\right)$ como en 2014 $\left(X^{2}=304,6 ; p<0,001\right)$. En el mismo período, se verificó que ser indígena consistió en factor de riesgo para el suicidio (2005: OR = 4,04; IC95\%=3,22-5,05 e 2014: OR=4,18; IC95\% = 3,51-4,98). El grupo de edad más afectado entre los indígenas brasileños en el período fue la de 15 a 19 años, seguida por la 
franja de 20 a 29 años. El estado de la federación que registró el mayor número de muertes fue Mato Grosso do Sul, seguido por el Amazonas. Los datos encontrados indican que estas comunidades están en situación peligrosa demandando así la implementación de políticas de salud mental que deberán priorizarlos, tener en cuenta sus peculiaridades y su derecho de autodeterminación.

\section{PALABRAS CLAVE}

Epidemiología; el suicidio; Población Indígena; Etnicidad.

\section{INTRODUÇÃO}

Comunidades indígenas seguem enfrentando problemas graves em todo o mundo: indígenas vivem menos, tem piores serviços de saúde e de educação e tem taxas de desemprego mais altas que a população em geral (UNITED NATIONS, 2009). A estes problemas somam-se outros como o tabagismo, as altas taxas de encarceramento, as altas taxas de alcoolismo, de abuso de drogas e de depressão (CERRON, 2014).

De tais problemas talvez o mais intrigante sejam as altas taxas de suicídio que vêm sendo verificadas nestas comunidades, principalmente quando se leva em conta que são os adolescentes e adultos jovens que mais tem recorrido a este ato desesperado (UNITED NATIONS, 2009).

Mundialmente este fenômeno tem se verificado até entre países desenvolvidos.

Na Austrália, em 2016, a taxa de suicídio entre jovens de 15 a 24 anos foi de 12.7/100.000 para a população total, enquanto para os jovens indígenas da mesma faixa etária foi de 39.2/100.000 (ARACY, 2018).

Nos Estados Unidos, a taxa de suicídio em 2005 verificada entre crianças de comunidades indígenas e entre nativos do Alasca com idade de 5 a 14 anos foi 2,6 vezes maior do que a taxa encontrada para a população americana. Entre adolescentes indígenas de 15 a 24 anos, a taxa encontrada foi 3,5 vezes maior que a taxa nacional para a mesma faixa etária (HUMMINGBIRD, 2011).

No Canadá, de 2009 a 2011, a taxa de suicídio foi de 11,3/100.000. Nas áreas de alta concentração demográfica dos Inuit, esquimós de regiões árticas, no mesmo período, as taxas de suicídio foram 6,5 vezes maiores que as nacionais. Nas áreas com altas concentrações de indígenas a taxa foi 3,7 vezes maior que a nacional, e entre Metis foi 2,7 vezes maior (PAN-CANADIAN..., 2018).

Na Noruega Ártica, uma coorte que acompanhou de 1970 a 1998 um grupo de 19.801 indígenas Sami encontrou significativo aumento de risco de suicídio entre eles. Encontraram ainda significativo aumento da mortalidade para os jovens Sami com idade entre 15-24 anos para ambos os sexos (SILVIKEN et al., 2006).

Países da América Latina têm convivido com o mesmo problema.

$\mathrm{Na}$ Colômbia, pelo menos 15 jovens dos povos Embera, Wounaan, Katio e Chami se suicidaram em 2003 e 2004, sendo que a população destas comunidades era de aproximadamente 3.000 indivíduos (taxa de suicídio de 500/100.000) ao tempo em que a taxa geral de suicídios na Colômbia foi de 4,4/100.000 em 2003, segundo estatísticas do governo (FORENO, 2004). 
O Brasil tem verificado historicamente taxas de suicídio consideradas baixas se comparadas com outros países, no entanto, também aqui os números relativos aos indígenas são piores e divergentes da população em geral (MARíN-LEON et al., 2012).

No Amazonas, de 2006-2010, a taxa foi de 18,4/100.000 o que é 4,4 vezes a taxa observada entre não indígenas no mesmo período para a população daquele estado da federação (SOUZA; ORELLANA, 2013). Entre indígenas do Amazonas, a faixa etária de 15-24 anos foi a que apresentou maiores taxas - 37,7/100.000. Entre não indígenas, a faixa de maior taxa foi a de 60 anos ou mais, com 14,9/100.000 (SOUZA; ORELLANA, 2013). Altas taxas de suicídio têm sido observadas entre os Sorowaha que residem na bacia do rio Purus no Amazonas. Durante o período de 1980 a 1995, o suicídio foi a maior causa de mortes, respondendo por $57,6 \%$ dos óbitos ocorridos no período. A maioria dos suicidas eram jovens de ambos os sexos (POZ, 2000).

No Centro-Oeste todos os casos de suicídio se deram em Mato Grosso do Sul (MARín-LEON et al., 2012), estado que possui a segunda maior nação indígena do Brasil com 66.963 índios, dos quais 40.245 são Guarani/Kaiowá. Ocorreram 410 suicídios nessa região de 2000 a agosto de 2008. A maioria dos suicidas eram homens, 65\% na faixa etária de 15 a 29 anos (GRUBITS et al., 2011).

Historicamente, porém, estes povos foram negligenciados nos censos demográficos de modo que os dados relativos eram em sua maior parte estimados, comprometendo assim, a confiabilidade dos estudos. Foi somente o Censo de 2010 que investigou estas comunidades, levantando dados mais precisos (IBGE, 2012a). Trouxe, portanto, a possibilidade de levantamentos mais fidedignos sobre 0 suicídio nestes grupos, condição essencial para a aferição da real gravidade da situação.

Este estudo objetivou levantar, de 2005 a 2014, o número de óbitos por suicídio no país, as taxas de mortalidade respectivas, as formas como estes óbitos se distribuem em cada etnia, entre os estados da federação e as faixas etárias mais atingidas. Visa, ainda, determinar associação entre etnia e mortalidade por suicídio, bem como se ser indígena no Brasil é fator de risco para tal mortalidade.

\section{METODOLOGIA}

Trata-se de um estudo epidemiológico do tipo transversal, utilizando-se como dados as respectivas frequências absolutas de suicídio em cada etnia, em 2005 e 2014, com vistas a identificar a distribuição geográfica, a faixa etária mais atingida e possíveis associações. 0 estudo foi com base populacional de todos os óbitos causados por lesões autoprovocadas e por autointoxicação, segundo a Classificação Internacional de Doenças, $10^{a}$ revisão, Códigos X60 a X84 (WHO, 2011).

A variável primária, definida como desfecho, foi o número de suicídios e bem assim seus coeficientes de mortalidade registrados no Brasil, ao tempo em que as variáveis explicativas foram: ano dos óbitos, faixa etária, estado da federação em que o óbito ocorreu e etnia (raça/cor autodeclarada). Dados demográficos obtidos por meio do Censo Demográfico 2010 do Instituto Brasileiro de Geografia e estatística (IBGE, 2012b). A partir destes dados, foram calculadas as estimativas populacionais do período intercensitário. Tais números, aliados aos números de óbitos por lesões autoprovocadas 
fornecidos pelo Sistema de Informações sobre mortalidade - SIM/DATASUS (BRASIL, 2017), subsidiaram a obtenção dos coeficientes de mortalidade por suicídio em cada raça/etnia respectivas.

Para comparar a mortalidade das populações estudadas foram calculadas taxas ajustadas por idade, considerando como população padrão a população total do Brasil, no sentido de ser reduzido o efeito das diferenças de estrutura etária dessas populações.

Os dados foram analisados pelo programa RStudio, versão 1.0.153 (RSTUDIO TEAM, 2015). Para a verificação de possíveis associações bem como a razão de chances para o referido agravo, utilizou-se as funções em R "chi-squared" e "proc glm, family=binomial”. Para tais análises, adotou-se um nível de significância de p-valor igual a 0,05.

A presente pesquisa foi autorizada pelo Comitê de Ética do Centro Universitário Tiradentes/UNIT/ AL, por meio do parecer de $n^{0} 2.033 .806 / 2017$.

\section{RESULTADOS E DISCUSSÃO}

No Brasil foram registrados no SIM/DATASUS 95.566 óbitos por lesão autoprovocada intencionalmente, no período de 2005 a 2014, revelando uma taxa média de mortalidade por suicídio de 4,97/100.000 para a referida década, ou seja, uma taxa relativamente baixa na mesma linha do que se vem apontando historicamente na literatura para o país (MARÍN-LEON et al., 2012).

A Tabela 1 apresenta a distribuição dos óbitos por suicídio entre as etnias referidas.

Tabela 1 - Número de óbitos por suicídio no Brasil entre pessoas de 10 anos ou mais de idade de 2005 a 2014

\begin{tabular}{cccc}
\hline Etnia & No. Óbitos por suicídio & \% & $\begin{array}{c}\text { Taxa média } \\
\text { (por 100.000) }\end{array}$ \\
\hline Brancos & 49.216 & 51,0 & 5,13 \\
Pardos & 35.456 & 37,0 & 4,37 \\
Negros & 5.102 & 5,3 & 3,72 \\
Indígenas & 935 & 0,9 & 10,50 \\
Amarelos & 399 & 0,4 & 1,90 \\
Não Informada & 4.458 & 4,6 & - \\
\hline
\end{tabular}

Fonte: MS/SVS/CGIAE - Sistema de Informações sobre Mortalidade - SIM e IBGE.

O número de óbitos verificado entre indígenas foi um dos menores, se comparado com as outras etnias. No entanto, quando considera a base de referência e seus respectivos coeficientes médios de mortalidade por suicídio no mesmo período, a situação se inverteu, passando os indígenas apresentar o grupo de maior risco de ocorrência do desfecho, ou seja, de registro de óbito por lesão autoprovocada voluntariamente (TABELA 1). 
Os dados da tabela 1 informam ainda que, assim como foi encontrado na Colômbia, Canadá, Austrália e Estados Unidos (UNITED..., 2009; PAN-CANADIAN..., 2018), no Brasil as taxas de mortalidade por suicídio verificadas entre indígenas, também, foram superiores à taxa nacional: a taxa de mortalidade entre indígenas, na verdade foi cerca de duas vezes maior que a taxa média nacional.

Quanto à distribuição geográfica, o SIM/DATASUS indica que o estado da federação que registrou o maior número de óbitos de indígenas por suicídio foi o Mato Grosso do Sul com 395 óbitos (42,2\% dos óbitos de indígenas), seguido pelo Amazonas, com 328 óbitos no período (35,0\% dos óbitos de indígenas). Roraima teve 62 óbitos e os demais estados, juntos, registraram 147 óbitos.

Estes dados advertem para a gravidade do problema no estado de Mato Grosso do Sul, considerando que a população de indígenas que lá habitava, 21.419 em 2014, era bem inferior ao número de indígenas habitantes no Amazonas (87.779, 10,7\% do total de indígenas também em 2014).

A faixa etária mais atingida entre os indígenas foi a de 15 a 19 anos na qual ocorreram 32,7\% dos óbitos, seguida de perto pela faixa de 20 a 29 anos, com 29,5\% dos óbitos segundo os dados pesquisados. Em 2010, os óbitos por suicídio verificados entre indígenas assim se distribuíram entre as faixas etárias (TABELA 2).

Tabela 2 - Óbitos por suicídio, população residente e taxa de mortalidade por suicídio no Brasil entre indígenas por faixa etária em 2010

\begin{tabular}{cccc}
\hline $\begin{array}{c}\text { Faixa Etária } \\
\text { (anos) }\end{array}$ & Óbitos & População & $\begin{array}{c}\text { Taxas } \\
\text { (por 100.000) }\end{array}$ \\
\hline $5-9$ & - & 109.104 & - \\
$10-14$ & 11 & 104.221 & 10,55 \\
$15-19$ & 28 & 90.160 & 31,06 \\
$20-29$ & 29 & 152.445 & 19,02 \\
$30-39$ & 18 & 115.441 & 15,59 \\
$40-49$ & 4 & 84.751 & 4,72 \\
$50-59$ & 1 & 59.139 & 1,70 \\
$60-69$ & 1 & 39.157 & 2,55 \\
$\geq 70$ & 0 & 32.256 & - \\
\hline
\end{tabular}

Fonte: MS/SVS/CGIAE - Sistema de Informações sobre Mortalidade - SIM e IBGE.

A taxa verificada entre os jovens indígenas de 15 a 19 anos é 31,05/100.000, ou seja, 8,7 vezes maior que a taxa obtida para esta mesma faixa etária, considerando toda a população brasileira (3,56/100.000). Aliás, o perfil etário da população indígena é diferente do perfil etário nacional em vários aspectos, consoante se pode observar comparando a Tabela 2 com a Tabela 3. Com efeito, no contexto nacional a faixa etária mais atingida é a de 80 anos ou mais, seguida pela faixa de 40 a 49 anos, cuja taxa é muito próxima da taxa para a faixa de 70 a 79 anos. 
Tabela 3 - Óbitos por suicídio no Brasil por faixa etária em 2010

\begin{tabular}{cccc}
\hline $\begin{array}{c}\text { Faixa Etária } \\
\text { (anos) }\end{array}$ & Óbitos & População & $\begin{array}{c}\text { Taxas } \\
\text { (por 100.000) }\end{array}$ \\
\hline 5 a 9 & 3 & 14.969 .375 & 0,02 \\
10 a 14 & 101 & 1.716 .6761 & 0,50 \\
15 a 19 & 605 & 16.990 .870 & 3,50 \\
20 a 29 & 2.210 & 34.349 .603 & 6,43 \\
30 a 39 & 1.996 & 29.633 .093 & 6,70 \\
40 a 49 & 1.842 & 24.842 .718 & 7,41 \\
50 a 59 & 1.240 & 18.416 .621 & 6,73 \\
60 a 69 & 719 & 11.349 .929 & 6,33 \\
70 a 79 & 461 & 6.305 .085 & 7,31 \\
$\geq 80$ & 246 & 2.935 .585 & 8,38 \\
\hline
\end{tabular}

Fonte: MS/SVS/CGIAE - Sistema de Informações sobre Mortalidade - SIM e IBGE.

Este resultado, também, foi semelhante ao que vem sendo encontrado na literatura para comunidades indígenas da Austrália, cuja taxa de suicídios entre jovens, no ano de 2016, foi cerca de três vezes superior à taxa verificada entre jovens de toda a população dos Estados Unidos (ARACY, 2018), onde em 2005 ela foi 3,5 vezes maior que a nacional observada entre jovens, (HUMMINGBIRD, 2011) e do Canadá (PAN-CANADIAN..., 2018). Observa-se que este fenômeno já foi visto na literatura em um estudo realizado na Noruega no período de 1970 a 1998 (SILVIKEN et al., 2006). Resultado similar foi encontrado para o período de 2006-2010 no estado do Amazonas, no qual foram encontradas diferenças significativas $(p<0,05)$ na variável idade entre indígenas e não indígenas que cometeram suicídio no referido estado (SOUZA; ORELLANA, 2013).

A explicação mais difundida sobre este comportamento suicida entre os indígenas o atribui a fatores culturais, especialmente o enfraquecimento e colapso das instituições culturais e a aculturação compulsória destes povos que se seguiu ao processo de colonização e modernização (CERRON, 2014; UNITED NATIONS, 2009; SOUZA; ORELLANA, 2013; THE FIRST..., 2012).

Poz (2000), ao analisar o caso dos Sorowaba, entende pela inadequação do modelo Durkheimiano para explicar o fenômeno e o atribui à ênfase excepcional que a sociedade sorowaha empresta às virtudes físicas e morais, sobretudo as que conotam a juventude. Segundo o referido autor, esta comunidade exige muito dos jovens quanto ao seu desempenho individual (força física, habilidade, disposição, beleza, controle da sexualidade, entre outros) o que os tornariam mais susceptíveis a eventuais desavenças e desgostos. Aliado a isso, a morte por suicídio no modelo cosmológico nesta comunidade, ocupa um patamar celeste superior ao alcançado por aqueles que morrem de velhice ou de picada de cobra. 
Enquanto Morgado (1991) entende que o fenômeno pode ser melhor explicado pela Hipótese do Recuo Impossível. Esta hipótese caracteriza-se pela ocorrência simultânea de dois tipos de sujeição máxima da pessoa: (1) total esgotamento de opção para recuar ou mudar, sem nenhuma possibilidade de território para os indígenas viverem, enquanto povo com identidade própria; e, (2) degradação extrema de condições universais de dignidade do ser humano, que independe da pessoa ser um indígena ou não; em outras palavras, liquidar o patrimônio material e cultural, corromper os costumes e aviltar a pessoa.

Ainda, segundo Morgado (1991), o fato de haver epidemia de suicídio entre indígenas de outros países não infirma sua hipótese, pois também nestes casos os índios se encontravam em limite de recuo e com chances escassas de inserção na civilização ocidental. Anota ainda que grupos humanos em condições de recuo impossível prescindem de representações específicas sobre a morte para virem a praticar ato de autoimolação.

Outros destacam que, no caso brasileiro, as regiões que ostentam as maiores taxas de suicídio estão próximas de fronteiras internacionais, convivem com tráfico de drogas e de armas, além de enfrentarem permanentes conflitos por terra (SOUZA; ORELLANA, 2013; CERRON, 2014).

Uma causa hipotética para este fenômeno proposta por Souza e Orellana (2013) seria a frustração dos jovens em perder seus meios tradicionais de subsistência, aliada à frustrante realidade que encontram após a conclusão do ensino médio, que é desemprego. Outra hipótese aventada pelos referidos autores seria a de que tais suicídios se devem ao consumo de bebidas alcoólicas, uma vez que a maior proporção de suicídios ocorreu nos dias de fim de semana. Há ainda estudos, suportando a hipótese de que o suicídio pode ter um caráter contagioso, de modo que a exposição de pessoas a esta experiência aumente o risco de ideação suicida, de tentativa e de morte por suicídio, especialmente entre adolescentes (SWANSON, 2013).

Há ainda estudos, suportando a hipótese de que o suicídio pode ter um caráter contagioso, de modo que a exposição de pessoas a esta experiência aumente o risco de ideação suicida, de tentativa e de morte por suicídio, especialmente entre adolescentes (SWANSON, 2013).

Embora no período estudado tenha sido entre os indígenas que se observou a maior variação de taxa de suicídio (reduziu de 22,37/100.000 em 2005 para 14,26/100.000 em 2014), em ambas as situações ser indígena configurou-se fator de risco para o óbito por suicídio (2005: OR = 4,04; IC95\%=3,22-5,05; $X^{2}=174,9 ; p<0,001$ e 2014: OR =4,18; IC95\% = 3,51-4,98; $\left.X^{2}=304,6 ; p<0,001\right)$.

\section{CONCLUSÃO}

O número de óbitos por suicídio entre indígenas foi menor que o verificado entre a maioria das outras etnias, perdendo apenas para os amarelos. No entanto, o coeficiente de mortalidade por suicídio de indígenas foi o dobro da taxa de suicídio da população geral do Brasil nos anos de 2005 e 2014 e nesses dois anos houve associação entre ser indígena e suicídio. A faixa etária mais acometida foi de 15 a 19 anos, sendo seguida pela faixa etária de 20 a 29 anos. 0 estado que registrou o maior número de óbitos foi Mato Grosso do Sul, seguido pelo Amazonas. 
Este quadro é lamentável por ser indicativo de outras mazelas como transtornos mentais graves, desemprego, desagregação social, além da crônica exclusão destas populações da sociedade brasileira em geral e da falta de assistência social, médica e educacional.

Altas taxas de suicídio podem, ainda, comprometer a subsistência da própria comunidade, pois, atinge jovens em idade reprodutiva, impactando negativamente a taxa de natalidade. Perdem-se assim vidas preciosas, variadas culturas e uma parte importante da história do país.

A situação se torna mais preocupante quando se leva em conta a subnotificação do campo raça/ cor nas declarações de óbitos no país. Mais de $4 \%$ dos óbitos registrados não informaram a etnia e é provável que haja muitos óbitos sem registro no SIM/DATASUS, o que sugere que a situação real seja ainda mais grave.

Os resultados encontrados, porém, já constituem evidências suficientes para justificar a necessidade de elaboração e implementação de políticas públicas voltadas a prevenção do suicídio nessa população.

Estas ações de saúde, contudo, para serem efetivas devem levar em conta as peculiaridades sociais, econômicas e culturais de cada etnia. Deverão ainda respeitar o direito de autodeterminação destas comunidades o que significa criar condições para a participação total e efetiva delas no planejamento e gestão dos sistemas de saúde, além da adesão ao princípio do livre consentimento, de ser previamente informadas dos programas e projetos de saúde a serem implantados.

\section{REFERÊNCIAS}

ARACY. The Australian Research Alliance for Children and Youth. The Wellbeing of Young

Australians. 2018. Disponível em: https://www.aracy.org.au/publications-resources/command/ download_file/id/361/filename/ARACY_Report_Card_2018.pdf. Acesso em: 14 jul. 2018.

BRASIL. Ministério da Saúde. DATASUS. Sistema de Informações sobre Mortalidade (SIM/SUS). 2017. Disponível em: http://tabnet.datasus.gov.br/cgi/deftohtm.exe. Acesso em: 21 out. 2017.

CERRON, I. T. et al. Suicídio Adolescente em Povos Indígenas. 3 Estudos. São Paulo: Arte Brasil Editora, 2014. Disponível em: https://www.iwgia.org/images/publications/0630_ SuicAdolescPovosInd_ALTA.pdf. Acesso em: 20 fev. 2018.

FORENO, J. In a land torn by violence, too many troubling deaths. New York Times. New York, 23 nov. 2004. Disponível em: https://www.nytimes.com/2004/11/23/health/psychology/in-a-landtorn-by-violence-too-many-troubling-deaths.html. Acesso em: 14 jul. 2018.

GRUBITS, S. et al. Suicidios de jóvenes Guaraníes/Kaiowás de Mato Grosso do Sul, Brasil. Psicol Ciênc Prof, v. 31, n. 3, p. 504-517, 2011. Disponível em: http://www.scielo.br/scielo.php?script=sci_ arttext\&pid=S1414-98932011000300006\&lng=en\&nrm=iso. Acesso em: 14 jul. 2018. 
HUMMINGBIRD, L. The Public Health Crisis of Native American Youth Suicide. NASN S Nurse, v. 26, n. 2, p. 110-114. 10 mar. 2011 Disponivel em: https://doi.org/10.1177\%2F1942602X10397551. Acesso em: 14 jul. 2018.

IBGE - Instituto Brasileiro de Geografia e Estatística. Censo Demográfico 2010. Características Gerais dos Indígenas - Resultados do Universo. Rio de Janeiro, 2012a. Disponível em: https://ww2. ibge.gov.br/home/estatistica/populacao/cens02010/caracteristicas_gerais_indigenas/default_ caracteristicas_gerais_indigenas.shtm. Acesso em 18 jun. 2018.

IBGE. Instituto Brasileiro de Geografia e Estatística. Censo Demográfico 2010. Resultados Gerais da Amostra. Rio de Janeiro, 2012b. Disponível em: http://prattein.com.br/home/images/stories/ Direitos_da_Criana_e_do_Adolescente/resultados_gerais_amostra.pdf. Acesso em: 2 nov. 2016.

MARÍN-LEÓN, L. et al. Suicide in Brazil, 2004-2010: the importance of small counties. Rev Panam Salud Publica, v. 32, n. 5, p. 351-359, 2012. Disponível em: https://scielosp.org/pdf/rpsp/2012. v32n5/351-359/en. Acesso em: 14 jul. 2018.

MORGADO, A. Epidemia de suicídio entre os Guaraní-Kaiwá: indagando suas causas e avançando a Hipótese do Recuo Impossível. Cad Saúde Públ, v. 7, n. 4, p. 585-598, 1991. Disponível em: https:// www.scielosp.org/pdf/csp/1991.v7n4/585-598/pt. Acesso em: 17 jul. 2018.

PAN-CANADIAN Health Inequalities Reporting Initiative. Key Health Inequalities in Canada: A National Portrait. Her Majesty the Queen in Right of Canada, as represented by the Minister of Healthy, 2018. Disponível em: https://www.canada.ca/content/dam/phac-aspc/documents/ services/publications/science-research/key-health-inequalities-canada-national-portrait-executivesummary/hir-full-report-eng.pdf. Acesso em: 18 jul. 2018.

POZ, J. Crônica de uma morte anunciada: do suicídio entre os Sorowaha. Rev Antropol, v. 43, n. 1, p. 89-144, 2000. Disponível em: http://www.scielo.br/scielo.php?script=sci_arttext\&pid=S0034$77012000000100004 \&$ lng=en\&nrm=iso. Acesso em: 19 jul. 2018.

RSTUDIO Team (2015). RStudio: Integrated Development for R. RStudio, Inc., Boston, MA URL http://www.rstudio.com.

SILVIKEN, A. et al. Suicide among indigenous Sami in Artic Norway, 190-1998. Eur J Epidemiol, v. 21, n. 9, p. 707-713, 2006. Disponível em: <https://doi.org/10.1007/s10654-006-9052-7>. Acesso em: 18 jul. 2018. 
SOUZA, M. L. P.; ORELLANA, J. D. Y. Desigualdades na mortalidade por suicídio entre indígenas e não indígenas no estado do Amazonas, Brasil. J Bras Psiquiatr, v. 62, n. 4, p. 245-252. 2013. Disponível em: http://repositorio.observatoriodocuidado.org/handle/handle/153. Acesso em: 17 jul. 2018.

SWANSON, S. Association between exposure to suicide and suicidality outcomes in youth. CMAJ. v. 185, n. 10, p. 870-877. Jul. 2013. Disponível em: http://www.cmaj.ca/content/185/10/870. Acesso em: 25 jul. 2018.

THE FIRST Nations Information Governance Centre. First Nations Regional Health Survey (RHS) Phase 2 (2008/10). National Report on Adults, Youth and Children Living in First Nations Communities. 2012. Disponível em: http://fnigc.ca/sites/default/files/First_Nations_Regional_ Health_Survey_2008-10_National_Report.pdf. Acesso em: 25 jul. 2018.

UNITED Nations. The State of the World's Indigenous Peoples. 2009. Disponível em: http://www. un.org/esa/socdev/unpfii/documents/SOWIP/en/SOWIP_web.pdf. Acesso em: 23 fev. 2018.

WHO - World Health Organization. International Statistical Classification of Diseases, Injuries, and Causes of Death. 10 rev. Genebra: 2011. Disponível em: http://apps.who.int/classifications/ icd10/browse/2016/en\#/X60-X84. Acesso em: 20 fev. 2018. 
1 Especialista em Direito Processual Civil; Graduada em Direito pela Universidade Federal de Alagoas - UFAL; Graduanda de Psicologia pelo Centro Universitário Tiradentes/ AL. Email: vilmajrcvictal@hotmail.com

2 Graduanda de Psicologia do Centro Universitário Tiradentes/AL. Email: biancataguiar@hotmail.com

3 Mestre em Nutrição pela Universidade Federal de Alagoas - UFAL; Professor Adjunto I, Curso de Psicologia do Centro Universitário Tiradentes - UNIT/AL.

Email: antoniofernando_jr@yahoo.com

4 Pós-doutor em Bioestatística pela Universidade de Lisboa-Portugal; Doutor em Zootecnia pelo Programa de Doutorado Integrado em Zootecnia-PDIZ, Universidade Federal Rural de Pernambuco - UFRPE/Universidade Federal da Paraíba - UFPB/Universidade Federal do Ceará - UFC; Professor Associado da Universidade Federal de Alagoas UFAL. Email: cyrorcjr@gmail.com

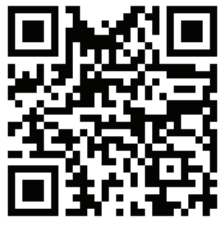

A autenticidade desse artigo pode ser conferida no site https://periodicos set.edu.br

\section{Como citar este artigo:}

Victal, Vilma Janaína Rios Cabral; Aguiar, Bianca Tenório; Xavier Júnior, Antônio Fernando Silva; Cabral Júnior, Cyro Rego. Suicídio e povos indígenas no Brasil. Interfaces Científicas Saúde e Ambiente, Aracaju, v. 7, n. 3, p. 49-60, abr. 2019. DOI: 10.17564/2316-3798.2019v7n $3 p 49-60$

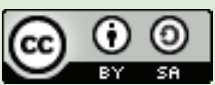

Este artigo é licenciado na modalidade acesso abertosob a Atribuição-Compartilhalgual CC BY-SA

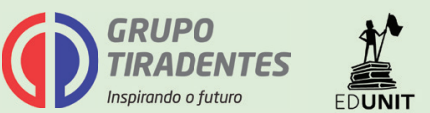

